\title{
A complementary algorithm for capacity enhancement of UMTS HSDPA
}

\author{
Jahangir Dadkhah Chimeh ${ }^{1 \mathrm{a})}$, Mohammad Hakkak ${ }^{2}$, Paeiz Azmi ${ }^{2}$, \\ and Hamidreza Bakhsi ${ }^{3}$ \\ ${ }^{1}$ Faculty of Engineering, Science and Research Branch, Islamic Azad University \\ Hesarak, Tehran, Iran \\ ${ }^{2}$ Faculty of Engineering, Tarbiat Modares University \\ Jalale Ale Ahmad, Tarbiat Modares University, Tehran, Iran \\ ${ }^{3}$ Faculty of Engineering, Shahed University \\ a)dadkhah@itrc.ac.ir
}

Abstract: DCH was solely used to convey data and voice traffic in the first releases of UMTS mobile systems. Apart from the properties of the traffic services, one OVSF code was permanently assigned to this channel. Then, to improve the system performance, DSCH and HS-DSCH channels were used in the next releases of the mobile systems for bursty traffic services. OVSF codes were only assigned to the channel in the data transfer time in these releases. In this paper by a multiplexer/scheduler, queue and a buffer we introduce a Traffic Model Scheduler (TMS) in layer two/RLC of HSDPA to reduce the OVSF code usage more in HS-DSCH.

Keywords: HS-DSCH, internet traffic, scheduler, OVSF

Classification: Wireless circuits and devices

\section{References}

[1] 3GPP TS 25.301 V6.7.0, Release 6, Radio interface protocol architecture.

[2] D. Wu and R. Negi, "Utilizing Multiuser Diversity for Efficient Support of Quality of Service Over a Fading Channel," IEEE Trans. Veh. Technol., vol. 54, no. 3, pp. 1198-1206, May 2005.

[3] M. Kazmi and N. Wiberg, "Scheduling Algorithms for HS-DSCH in a WCDMA Mixed Traffic Scenario," 14th IEEE Proc. Personal, Indoor and Mobile Radio Communications, 2003. PIMRC 2003, vol. 2, pp. 1485-1489, Sept. 2003.

[4] 3GPP TS 25.321, version 7.0.0 Release 7, "Medium Access Control (MAC) Protocol Specifications," 2006.

[5] J. D. Chimeh, M. Hakkak, P. Azmi, and H. Bakhshi, "Traffic Capacity of UMTS System in Reverse Link," Wireless Personal Commun., Springer, vol. 51, no. 2, pp. 303-316, Oct. 2008.

[6] J. D. Chimeh, M. Hakkak, and P. Azmi, "Internet Traffic Modeling and Capacity Evaluation in UMTS," Int. J. Hybrid Inf. Technol., vol. 1, no. 2, pp. 109-120, April 2008.

[7] 3GPP, R7, TS 25.322, "Radio Link Control (RLC) protocol specification," 


\section{Introduction}

In the ordinary HSDPA, OVSF code is assigned to a user when a traffic service is going to be transmitted, i.e. only one traffic service is handled by one RLC at an instant [1]. This may result in OVSF code shortage.

There are two multiplexer/scheduler in Layer 2/MAC of HSDPA. C/T MUX is in MAC-d and is responsible for multiplexing of logical channels. Beside that in MAC-hs there is a scheduler which selects the users according to their quality of channels. Many authors have worked on these algorithms to improve its performance $[2,3]$.

In these algorithms the payload (traffic packets) is treated as a black box and this fact degrades the performance of them because the traffic model parameters including traffic packet sizes, arrival times, inter-arrival between packets, etc. affect the throughput.

In TMS some different traffic services can be handled by one RLC. In other word, TMS is a real-time algorithm which handles different traffic types for a user when applications are in run. Thus in this paper we tried to make a change in the Layer 2/RLC to improve UMTS performance.

On the whole, to challenge the code shortage, there are two methods by the names of code reassignment and a clever algorithm for assigning new codes. In this paper we limit the OVSF usage by TMS. Thus we introduce the new algorithm in section 2 . In section 3 we pay attention to the simulation and results, in section 4 we study adaptation with the standard and finally we draw the conclusion.

\section{Suggested algorithm}

HS-DSCH transport channel is used for conveying the Internet traffic in HSDPA/3GPP in the downlink. This channel is used for time multiplexing of the different bursty traffic services such as www and email traffic services.

Suppose some applications are running simultaneously. The generated packets must be transmitted one after the other or simultaneously. Since it isn't possible to transmit the packets simultaneously in HSDPA and to handle the traffic services of simultaneous services, HSDPA uses some queues in the MAC layer [4].

The suggested TMS algorithm in this paper is a complementary real-time scheduler/multiplexer in layer 2/RLC which is implemented by a specific architecture, i.e. this algorithm is a real time Internet traffic packet multiplexer which operates before constituting the HS-DSCH. Here, we assume a user either runs one application several times or some different applications simultaneously, then the related data are downloaded at the same time. In other words we have assumed the aggregated traffic in the Node-B is constituted from different applications and directed to a user in downlink. 
Fig. 1 depicts streams of packets of $n$ applications according to the presented traffic model in [5] and [6]. Rectangles represent packets which are flowing to the output buffer sequentially. Multiplexer/scheduler sends out each user's packet according to its arrival time. For example packet A arrives first, then packet $\mathrm{b}$ arrives. Thus they go to the lower layers one after the other. Packet $\mathrm{C}$ arrives before packet $\mathrm{D}$, thus they have overlapped. Consequently while packet $\mathrm{C}$ is going to the lower layer packet $\mathrm{D}$ should be entered the proposed queue and so on. This queue is of the FIFO type in which the first input packet will be sent out first.

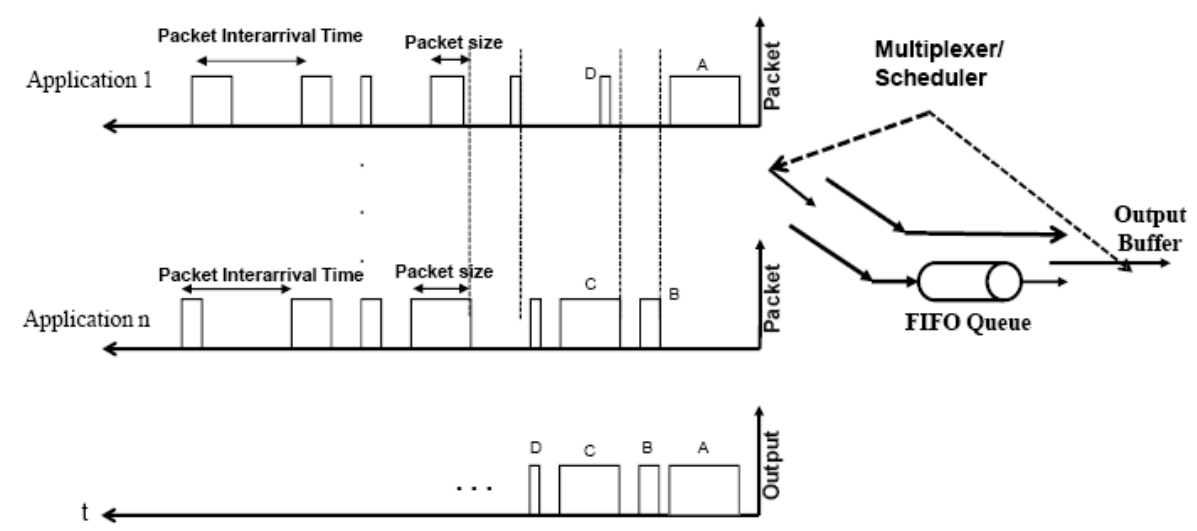

Fig. 1. Traffic Arrival and suggested TMS model in Node$\mathrm{B}$

If a packet encounters a full queue while entering it, blocking or packet less happens. We have also computed the maximum settling time of the packets of each application in the queue by name of transmission delay.

\section{Simulation and results}

Fig. 2 depicts different parts of the simulator and their interconnections which we describe each as follows.

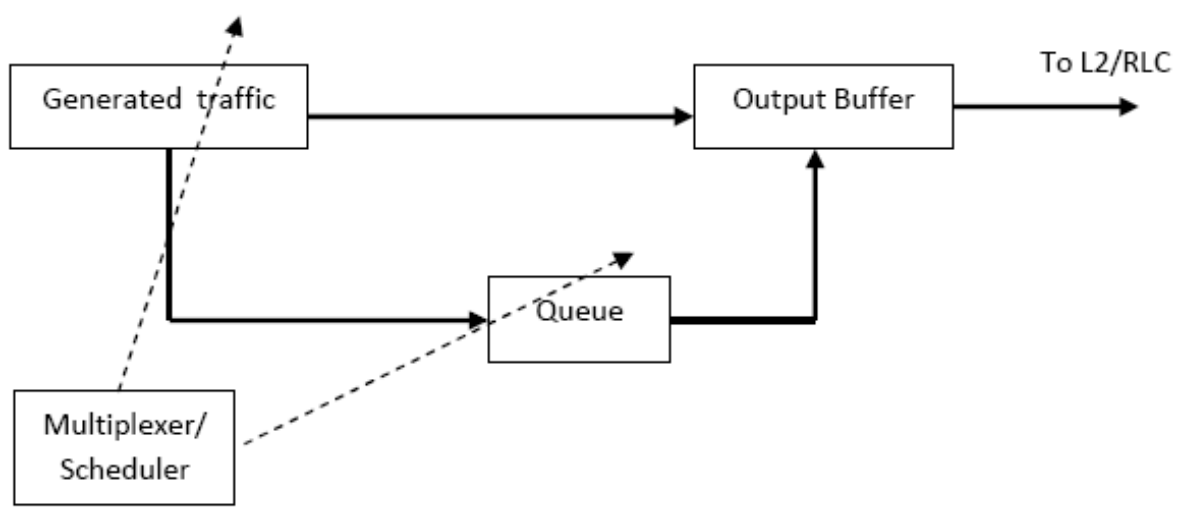

Fig. 2. Overview of different parts of the TMS simulator 
traffic services of a user according to the model presented in $[5,6]$ and the requested data rate.

The program includes an internal loop and an external loop. Internal loop is the main program that includes all the algorithm sections except queue manipulations and the external loop is responsible for it. Indeed external loop is responsible for the queue and sending/entering the data out/in.

We may run the program in two methods. Firstly, beginning with the queue length zero, user encounters the blocking. Each time the user encounters the blocking, the external program runs and the queue length increases. In the next run of the internal program we read the blocking parameter again and continue the above procedure till any packet will not be blocked. Secondly, we can also run the program based on the requested blocking rate, then we will find the queue length.

We first evaluate the performance of the proposed TMS model and algorithm. To evaluate them we computed the blocking rate and the delay and plotted them. We assumed 7 www and 3 email Internet applications enter the system according to the Poisson process with parameter 8 . We also assume output link rate is $64 \mathrm{kbps}$. The parameters of the generated traffic services are shown in Table I.

Table I. The parameters of services in the simulation [6]

\begin{tabular}{|c|c|c|c|c|c|}
\hline & $\begin{array}{c}\text { No. of } \\
\text { packet } \\
\text { calls per } \\
\text { session }\end{array}$ & $\begin{array}{c}\text { Inter-packet } \\
\text { call Arrival } \\
\text { time or } \\
\text { Reading } \\
\text { time calls (s) }\end{array}$ & $\begin{array}{c}\text { No. of } \\
\text { packets } \\
\text { per } \\
\text { packet } \\
\text { calls }\end{array}$ & $\begin{array}{c}\text { Packet } \\
\text { size }\end{array}$ & $\begin{array}{c}\text { Inter-Arrival } \\
\text { time between } \\
\text { packets (s) }\end{array}$ \\
\cline { 2 - 6 } & 5 & 120 & 25 & $\begin{array}{c}\text { Pareto } \\
(\mathrm{K}=81.5, \\
\alpha=1.1)\end{array}$ & 0.067 \\
$\begin{array}{c}\text { WWW } \\
\text { Traffic }\end{array}$ & 2 & 90 & 15 & $\begin{array}{c}\text { Pareto } \\
(\mathrm{K}=81.5, \\
\alpha=1.1)\end{array}$ & 0.067 \\
\hline $\begin{array}{c}\text { Email } \\
\text { Traffic }\end{array}$ & 2 & & & & \\
\hline
\end{tabular}

We used MATLAB for simulation. Fig. 3 depicts the blocking rate and maximum transmission delay of each application respectively.

As we see in Fig. 3 (a) the maximum blocked packets in queue length zero is $48 \%$. Blocking rate decreases while the queue length increases and at length 57 it approaches to $0 \%$. We see that for 10 applications and the queue length 35 , the blocking is less than $2 \%$ which is adequately acceptable. Fig. 3 (b) depicts that the maximum transmission delay varies between $0.66 \mathrm{~s}$ and $10.8 \mathrm{~s}$. Thus mean delay time is $3.06 \mathrm{~s}$. As it was shown we transfered the traffic of ten users by only one OVSF code with a reasoable blocking and delay. Thus, this is a very good benefit for the new system.

\section{Conclusion}

Using a TMS module including a scheduler, a queue and a buffer we gained a suitable blocking rate and delay and could reduce the OVSF codes several 


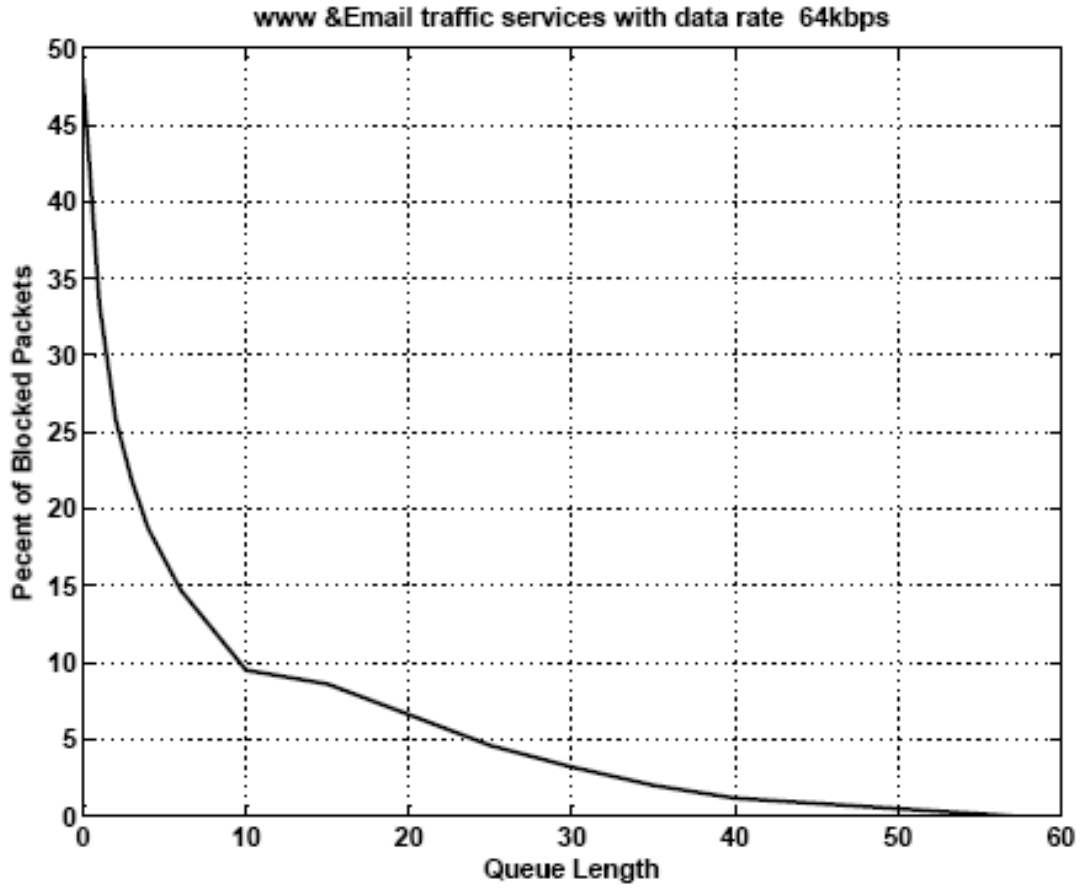

(a)

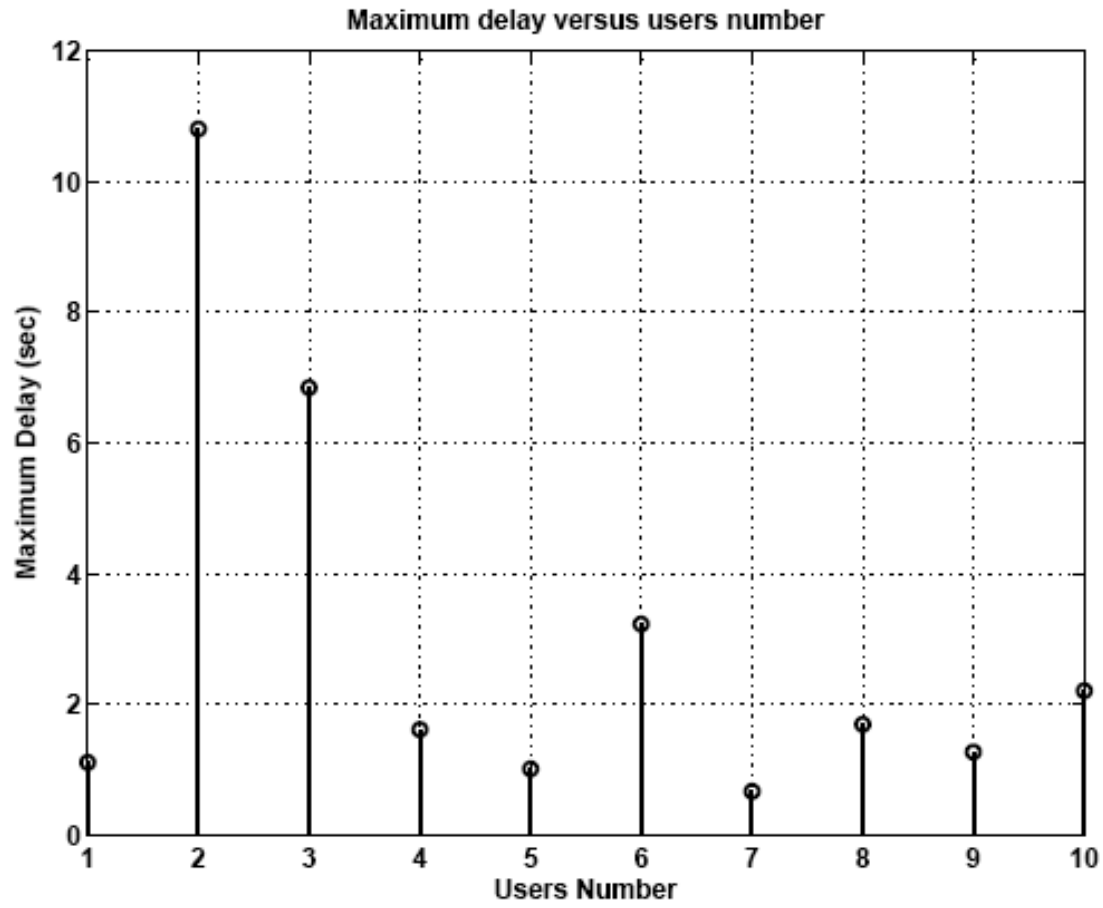

(b)

Fig. 3. Evaluation of TMS module

times more than the ordinary HSDPA case. Modifications were made on the top of the RLC. We should note that TMS is for traffic services of only one user in the downlink. 